\title{
A Study on Monitoring and Control Architecture for Smart Lighting System in IoT Environment
}

\author{
Hee-Dong Park1), Song-Gang Kim²)
}

\begin{abstract}
With the development of Information Technology(IT), smart lighting devices in the homes, office buildings and factories are evolving from the existing visual elements to non-visual elements in terms of functional and cognitive ability. In addition, these devices are utilizing monitoring functions through various environmental sensors. This paper suggests a monitoring and control architecture, and a frame structure for smart lighting system using wireless network toward Internet of Things(IoT) applications. The network can be organized as hierarchical architecture which includes physical devices, radio access modules connected as tree topology, and mobility service to interface internet for remote control and management. Using mobile devices that use ISM(Industry, Science, Medical) band, high-speed data transmission is possible; system status can be obtained through periodic status reporting from nodes; and request/reply structure for light device control is provided. Event reporting can be supported by frames and processing functions in main controller. Therefore, more efficient and low cost smart lighting system can be realized using this architecture.
\end{abstract}

Keywords : Smart Lighting Architecture, Monitoring and Control Protocol, Hierarchical Interface, Radio Tree Topology, Frame Structure, Internet of Things, Sensor Monitoring

\section{Introduction}

Currently, smart devices are becoming more and more in-demand with the emergence of smart home, smart farm, smart factory, smart building, and smart city, leading to changes in the trend of life. Lighting system refers the use of natural light to capture daylight and man-made light sources such as electric lamps and lighting equipments. The traditional concept of revealing darkness has gradually evolved into automatic control lighting according to the surrounding environment of people or some objects. In addition to energy saving, the smart

Received(April 25, 2019), Review Result(1st: May 20, 2019, 2nd: July 15, 2019), Accepted(September 10, 2019)

1) (Professor) 10279 Dept. Smart IT, Joongbu Univ., Dongheon-ro, Deogyang-gu, Goyang, Gyeonggi-do, Korea

email: hdpark@joongbu.ac.kr

2) (Professor, Corresponding Author) 10279 Dept. Smart IT, Joongbu Univ., Dongheon-ro, Deogyang-gu, Goyang, Gyeonggi-do, Korea

email: kimsg@joongbu.ac.kr 
lighting technology is now becoming important for efficient maintenance and easy expansion of lights, and also minimizing carbon emissions and light pollution.

Smart lighting has become a new technology, for example, for householder to control remotely the cooling and heating functions with air conditioning features using network interface. This feature provides saving energy and can also offer comfort and convenience of residence. And smart lighting also includes using sun light to reduce the use of electric lighting and the concept of turning off the lights when people goes out. Smart lighting is aimed at eco-friendly system that minimize energy consumption, and technically making remote control with wired/wireless communication, sensor control and data processing to provide the most stable and economical lighting control solution. It has developed into various types of applications such as streetlight or factory light control and home mood control, to take advantage of customized light control, energy saving and efficient maintenance for user's requirements.

Smart lighting control systems include integrated communication interfaces with internet connection approach which supports sensor inputs and control outputs using active devices for smart application and management. On using both indoor and outdoor environments, lighting control systems are becoming smarter in almost all fields including home, industry and office spaces, so can represent monitoring and control functions graphically when needed. Recently, various lighting control technologies have been proposed based on Internet of Things, which requires complicated and expensive devices and control systems.

There exists many studies on lighting systems, such as on the lighting and energy saving services over the IoT as well as traffic in smart city[1], lighting control techniques using DALI(Digital Addressable Lighting Interface) protocol[2], introduction of various existing communication protocols and network systems for lighting with the network topologies, link frame structure, protocol stack such as BACnet (Building Automation and Control Networks), CAN (Controller Area Network), DALI, and ZigBee[3]. In the study of Roy et. al.[4], it proposed an extended addressing scheme of ZigBee which supports all the original addressing scheme with applying the same algorithm for table-free routing scheme. In a study of lighting control using ZigBee[5], the LED control module was implemented using Zigbee protocol so that LED brightness can be changed according to the ambient environment. Also there exists a study to develop a communication module[6], which combines two communication environments, ZigBee in USN (Ubiquitous Sensor Network) and PLC (Power Line Communication) in power line, so supporting wired and wireless communication interface with integration protocol, and implemented network devices for wired and wireless network and 
control system software.

In the studies of Deepanshu et. al.[7] and Isah et. al.[8], design of energy saving technique for streetlight control and implementation of an automatic light control was described. Emi et. al. propose a concept on Internet of Lights (IoL) and describe the highlights of the important benefits and also the considerations to face in the future towards IoL[9]. To solve these challenges and meet specific requirements of lighting networks, an IoL reference architecture, Open AIS (Architecture for Intelligent Solid State Lighting Systems), has also been proposed. A study have confirmed that automatic lighting systems which have some environment sensors and individual controls are appropriate for office environments and can save a lot of energy compared to the conventional lighting system, even when the installed lighting density of the lighting system is higher[10].

From the viewpoint of energy saving, smart lighting control becomes important, and it requires a stable and convenient structure which can utilize the existing system or can improve the system at low cost. This paper proposes in section 2 a monitoring and control architecture for smart lighting system using wireless network toward Internet of Things applications, and also a design of communication protocol for use in a tree topology wireless network to monitor and transmit the environment data. Section 3 concludes and summarizes the study and recommendations for future research.

\section{Architecture, Frame and Protocol}

\subsection{Architecture}

There are various configurations to connect lighting control devices, and each depends on factors such as configuration topology, application goal, connection type and limitations. In particular, topology and communication protocols for control and monitoring the smart lighting devices are very important because these are related to the flexibility and reliability of system design, where two-way wireless communication systems are becoming essential for network modification and device mobility. This means that minimal changes to the physical wiring of existing systems is important. Network system using wireless devices that use $2.4 \mathrm{GHz}$ ISM band frequency can provide efficient system configuration by providing multiple signal path functions with extended range. Recently the rapid increasing uses of these bands have been applied in short-range, wireless low energy communications systems, since these bands are approved for devices or systems that can be used as defined by ITU (International 
Telecommunication Union) Radio Regulations.

In this study, the structure for remote monitoring and controlling the smart lighting equipment uses existing Wi-Fi or Internet, and low-cost tree-topology wireless network using ISM band frequency. The communication device used is the RF (Radio Frequency) module from Nordic, which enables wireless communication at the ISM frequency and is low cost but provides good performance. The structure for monitoring and controlling the smart lighting system is shown in [Fig. 1].

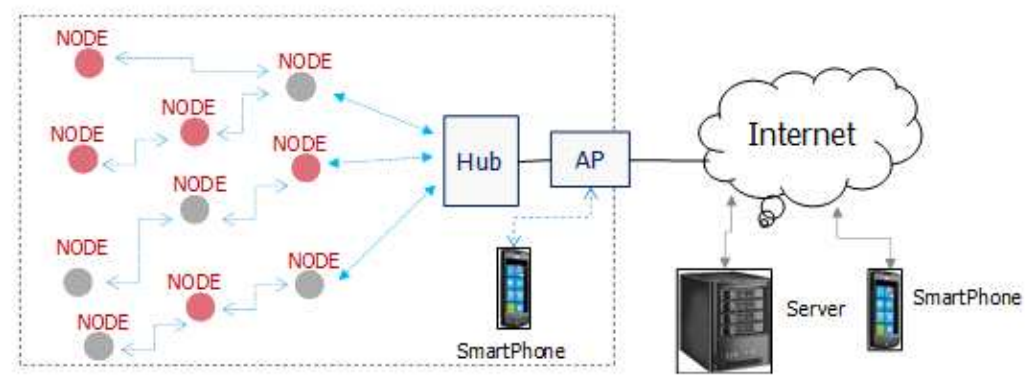

[Fig. 1] System Interface for Monitoring and Controlling the Smart Lighting Control

This structure can make environment monitoring and lighting control of devices in building or factory from a remote place through Internet connection, and provides such features to personal computers or mobile phones. By utilizing the broker function of IoT protocol on a server, statistical data and analysis can be done and provided to the users through collected data and big-data processing. The RF module supports up to 125 channels with 2.4 to 2.525 $\mathrm{GHz}$ frequency band, therefore interference with peripheral devices can be accordingly avoided. The data transmission speed is from 256Kbps to 2Mbps which supports enough bandwidth for monitoring and control data transmission. [Fig. 2] shows the structure of a tree-topology wireless network using RF devices.

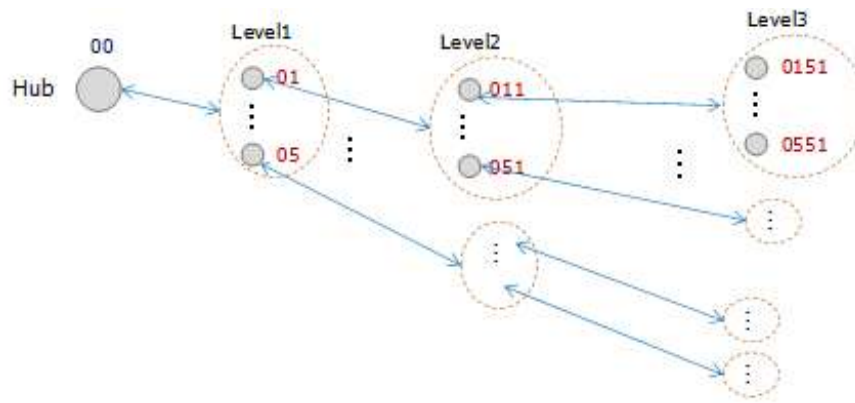

[Fig. 2] Structure of Tree-topology Wireless Network using RF Modules 
RF nodes in this tree network are linked by limited addresses granted as pipes. Each node can communicate to six nodes on six addresses, therefore each node has a parent address and five child addresses, so make a tree topology. Each node can communicate directly with its parent and children nodes. If a node want to send messages to a node except parent or children nodes, the message must be routed through the tree network. Routing of message is processed invisibly to the user, by the network layer function. The network addresses should be assigned before installation according to the physical layout of the network. All messages from each node will route to the destination automatically as required. For this routing operation, users will not consider routing in detail but simply construct a header containing the destination address, and the network layer protocol will forward it from node to next node[11]. The network structure with these operations can be expressed in a hierarchical architecture as shown in [Fig. 3].

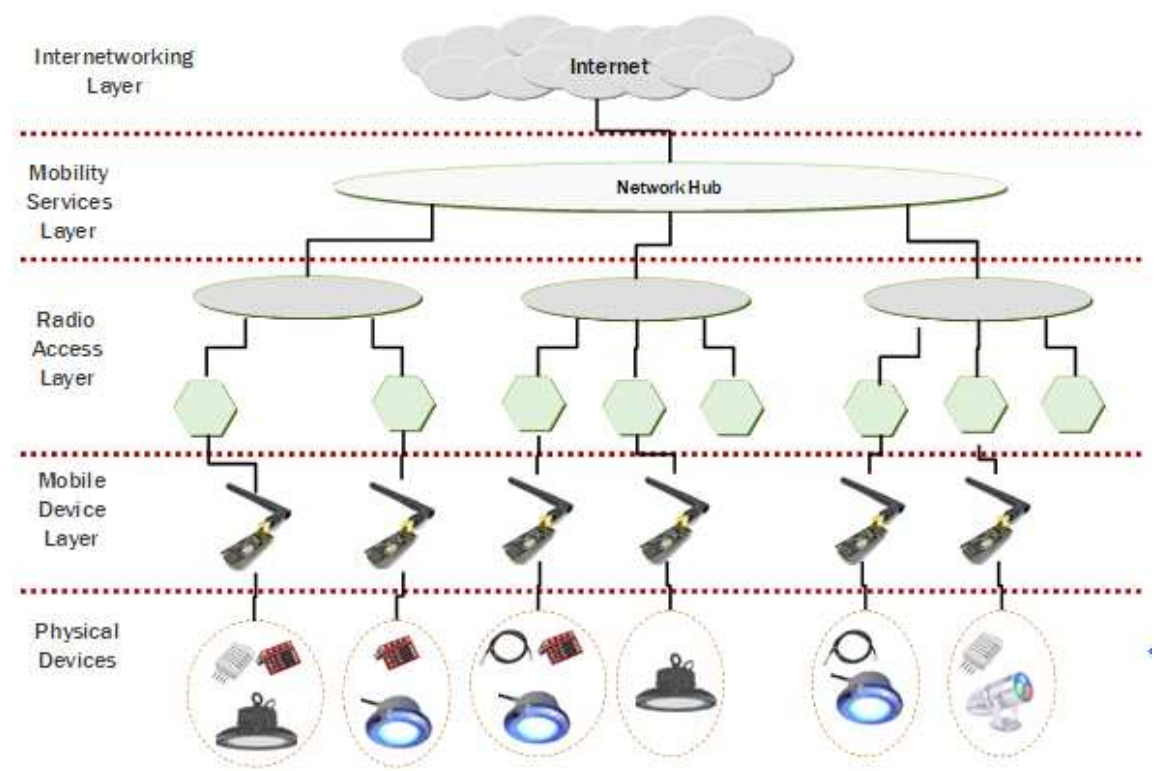

[Fig. 3] Hierarchical Architecture of Layered Interface for Smart Lighting Wireless Network

The physical devices are connected to an RF module in the mobile device layer, which is interfaced to the radio access layer for each group. Devices in radio access layer are connected each as tree topology to form a wireless network for smart lighting devices. A hub is interfaced to a mobility service layer which provides an internet connection to enable remote control and management. For routing data between parent and child nodes, the network uses built-in acknowledgement and retry functions of the chip to prevent data loss. When payloads need to be delivered to other nodes, routing processing will also be needed. Routing is 
controlled using built in acknowledgement requests and network layer acknowledgement replies implemented by protocol software. This scheme makes all routing nodes to transfer payloads quickly, while only the final routing node will confirm message delivery and send an acknowledgement back to the source node.

\subsection{Frame Structure}

Messages for status monitoring and light control can support periodic reporting of node status or sensor data, and also support control commands sent from the hub and corresponding responses from the nodes. [Fig. 4] shows the payload format and the frame inside for the 16 octets payload size.

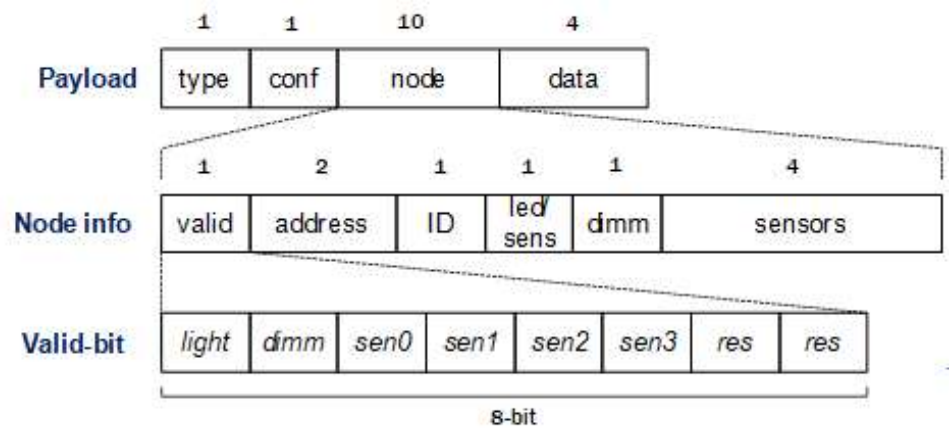

[Fig. 4] Frame Format of Payload

From [Fig. 4], up to four sensors, such as temperature, motion, CDS and etc. can be supported per node. Packet length is 16 bytes, therefore the influence on the network traffic is not heavy. Each field of a frame is shown in [Table 1].

[Table 1] Frame Fields and Description for Message Flow

\begin{tabular}{|c|c|l|}
\hline Type & Field & \multicolumn{1}{c|}{ Description } \\
\hline \multirow{4}{*}{ Payload } & type & Type of payload frame (Command/Request/Reply/Report) \\
\cline { 2 - 3 } & conf & Value for configuration \\
\cline { 2 - 3 } & node & Node information \\
\cline { 2 - 3 } & data & Auxiliary information (timestamp, sequence number) \\
\hline \multirow{4}{*}{ Node info } & valid & Bit-field enable(light, dimming, sensors) \\
\cline { 2 - 3 } & address & Node address \\
\cline { 2 - 3 } & ID & User index for operation \\
\cline { 2 - 3 } & led/sens & Bit-field(Light status and number of sensors) \\
\cline { 2 - 3 } & dimm & Dimming value (0 - 255) \\
\cline { 2 - 3 } & sensors & Value of configured sensors \\
\hline
\end{tabular}




\subsection{Protocol and Flow}

The communication protocol based on the tree-topology wireless network for smart lighting system monitoring and control can provide the functions of periodic monitoring by restoring the previous state and periodic reporting information about lighting condition and surrounding environment, and demand monitoring should also be supported. It also supports more intelligent monitoring and processing functions by allowing each node to immediately notify when an event occurs, such as security, motion detection and high temperature. In order to control the smart lighting system remotely using smart phone or PC via Internet, various types of message to a hub are defined through a computer server for this system depending on the connection stage, system configuration and operating period. The overall message flow between nodes, hub and clients is as follows:

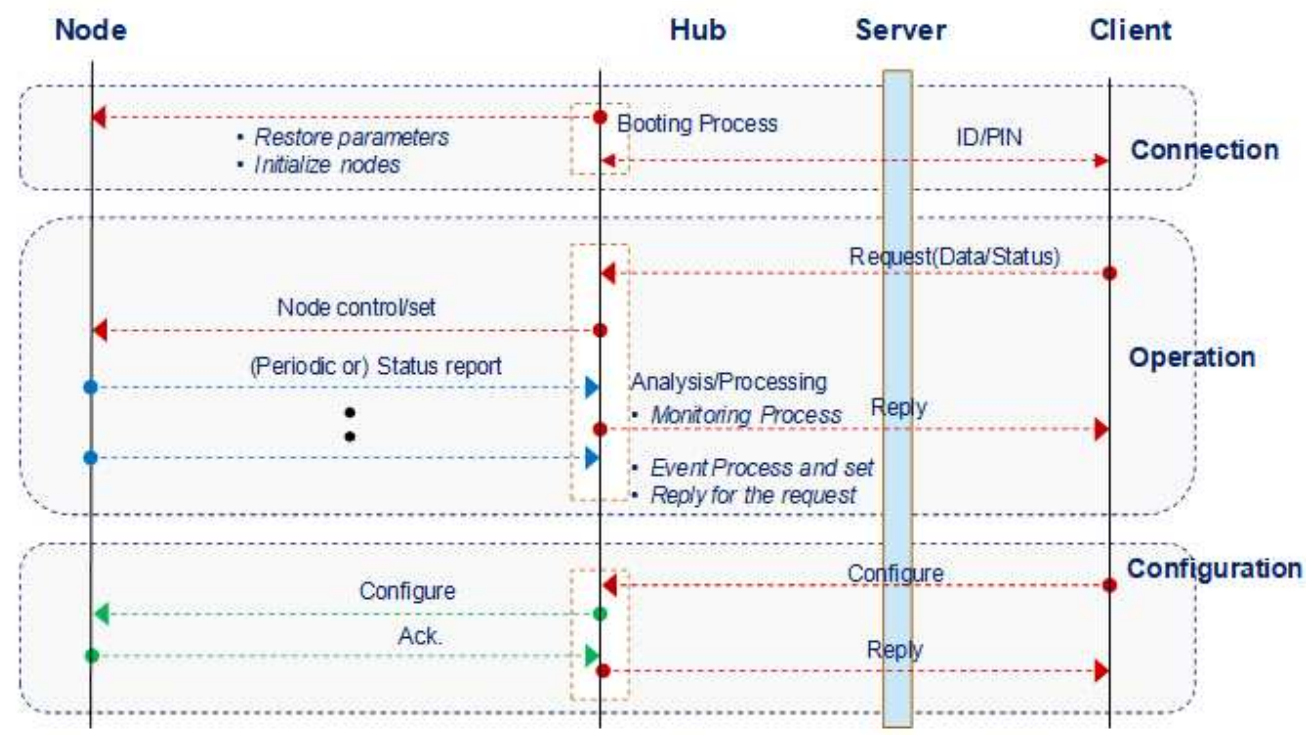

[Fig. 5] Status and Control Message Flow of Smart Lighting System Control

The hub stores information necessary for operation such as configuration and node data in the non-volatile storage, and at boot process, it extracts initialization data from storage and sets parameters to operate. That is, after reading the stored configuration data and initialize hub and node parameters based on the fetched data, then the appropriate nodes are initialized by performing the setup request protocol from the last storage data of each node. Additional hub storage data includes the SSID and password of the available AP, user ID and its password, but the server address and port number cannot be changed by the user to prevent non-allowed 
server access.

The hub processes periodic data from nodes and stores the node state in non-volatile memory when changed. To reduce traffic, the hub does not reply for the reporting information from nodes, and also does not request the responses to the node for normal control messages. In particular, if the hub receives no status report from the valid node for a certain period of time, it changes the node as invalid and informs the administrator. If the hub finds status report for an invalid or a new node, the node status in hub storage will be changed or allocated as a new node. For the lighting system configuration, the required functions for system operation are adding, modifying, and deleting nodes, which could be processed on the server and the hub, and it affects the initialization process of node with system boot time. The configuration results are stored in local storage of hub and client for future use.

Monitoring functions include status reporting and sensor data of nodes, which should be able to configure data type to transfer or reporting interval for each node. The status reporting interval of a node may be relatively long, but not for sensor data reporting. Because small packet loss in a smart lighting network is not serious to system operation, requesting retransmission or connection confirmation can be optional to reduce network traffic.

The protocol for controlling smart lighting system is based on the request/response message exchange structure, and supports the frame operation for the event processing. In the case of node or lighting control, various request types are supported and responses of each type should be received from the corresponding node. Otherwise, the state of each node will be confirmed by retransmission protocol. By using our proposed architecture, it is possible to provide smart management and monitoring functions for nodes and hub through cooperation with IoT protocol server. To provide intelligent system service, secured connection through user authentication, configuration of nodes from smart phone or user PC, data management and statistical function through big data processing should also be supported.

\section{Conclusion}

This paper describes an architecture of monitoring and control protocol for smart lighting system using wireless network to IoT applications. The network can be organized as hierarchical architecture which includes mobile device layer with physical devices, radio access layer connected as tree topology, and mobility service layer with internet connection to enable remote control and management. Using mobile devices that use ISM band, high-speed data transmission is possible, system status can be obtained through periodic status reporting from 
nodes, and specific light control using request/reply structure are also provided. Event reporting can be supported by defining frames and processing functions in main controller, so more efficient and low-cost smart lighting system can be realized using this protocol.

As the competition of wireless standards and protocols is evolving, developing and manufacturing of lighting system need to adapt their results to commercial and industrial lighting market when it is combined with the smart network and intelligent lighting control systems. A new Bluetooth mesh network supports complex, reliable, many-to-many communication between connected devices so that any device in the network can communicate with other device in the network. It is recommended for future work to use Bluetooth mesh which has full stack solution and spanning radio communications at the bottom of the stack and smart lighting applications at the top.

\section{References}

[1] B. Montgomery, Future Shock: IoT benefits beyond traffic and lighting energy optimization, IEEE Consumer Electronics Magazine, (2015), Vol.4, No.4, pp.98-100, DOI: 10.1109/MCE.2015.2463313

[2] DALI Standard, International Electrotechnical Commission IEC 62386, Available online: https://www.digitalilluminationinterface.org/dali/standards.html, May 30 (2019)

[3] S. G. Varghese, C. P. Kurian and V. I. George, A study of communication protocols and wireless networking systems for lighting control application, 2015 International Conference on Renewable Energy Research and Applications (ICRERA), (2015), November 22-25; Palermo, Italy, DOI: 10.1109/ICRERA.2015.7418618

[4] U. K. Roy, P. Bhaumik, Enhanced ZigBee tree addressing for flexible network topologies, Proceedings of 2015 Applications and Innovations in Mobile Computing (AIMoC), (2015), February 12-14; Kolkata, India, DOI: $10.1109 /$ AIMOC.2015.7083822

[5] Young-Ho Jang and Hwan-Yong kim, Implementation of a LED light control module using ZigBee, Journal of Korea Academia-Industrial cooperation Society, (2012), Vol.13, No.10, pp.4740-4744, DOI: 10.5762/KAIS.2012.13.10.4740

[6] Un-Seon Lee, Tae-Jin Park, Man-Gon Park, Implementation of Integrated Interface based on Wire and Wireless Dual Network for Ensuring the Reliability of Intelligent LED Lighting System, The Transactions of The Korean Institute of Electrical Engineers, (2014), Vol.63, No.2, pp.306-312, DOI: 10.5370/KIEE.2014.63.2.306

[7] Deepanshu Khandelwal, Bijo M Thomas, Kritika Mehndiratta, Nitin Kumar, Sensor Based Automatic Street Lighting system, International Journal of Education and Science Research Review (IJESRR), (2015), Vol.2, No.2, pp.24-27.

[8] Isah Abdulazeez Watson, Oshomah Abdulai Braimah, Alexander Omoregie, Design and Implementation of an 
Automatic Street Light Control System, International Journal of Emerging Technology and Advanced Engineering (IJETAE), (2015), Vol.5, No.3, pp.336-340.

[9] Emi Mathews, Salih Serdar Guclu, Qingzhi Liu, Tanir Ozcelebi and Johan J. Lukkien, The Internet of Lights: An Open Reference Architecture and Implementation for Intelligent Solid State Lighting Systems, Energies, (2017), Vol.10, No.8, p.1187, DOI: https://doi.org/10.3390/en10081187

[10] A. D. Galasiu, G. R. Newsham, Energy savings due to occupancy sensors and personal controls: a pilot field study, Lux Europa 2009, 11th European Lighting Conference, (2009), September 9-11; Istanbul, Turkey, pp.745-752.

[11] https://tmrh20.github.io/RF24Network/Tuning.html, May 20, (2015) 